\title{
ADAPTIVE EXPERT SYSTEM FOR CALCULATION OF REAL EAR INSERTION GAIN FOR DIGITAL HEARING AID
}

\author{
${ }^{1}$ Sadagopan Rajkumar, ${ }^{2}$ S. Muttan and ${ }^{3}$ Balaji Pillai \\ ${ }^{1}$ Department of ECE, Faculty of Information and Communication Engineering, \\ Kings Engineering College, Chennai-602117, India \\ ${ }^{2}$ Department of ECE, Faculty of Information and Communication Engineering, \\ CEG, Anna University, Chennai-600025, India \\ ${ }^{3}$ Department of otolaryngology, ENT Consultant-The Apollo Clinic, HSR Layout, Bangalore, India
}

Received 2013-07-01, Revised 2013-08-08; Accepted 2013-08-16

\begin{abstract}
The various gain calculating formulae for digital hearing aids is analyzed for different subjects to find out suitable adaptive gain formula for most successful gain recommendations. The object of the work is to design and develop an adaptive expert system, which could be effectively used to perform screening tests to identify the level of hearing impairment and recommend suitable gain suggestions for frequency bands of digital hearing aid. Initially, the design requirements for a digital hearing aid are being arrived by using the standard gain formulae followed such as National Acoustic Laboratory Revised (NAL-R) and Prescription of Gain Output (POGO II). The test is carried with 272 subjects aging from 25 to 72 and 221 male and 51 female. Of which gain is recommended for 127 subjects with the standard gain formula. When the recommendations are verified for satisfaction among the hearing aid users only 28 received satisfaction with NAL-R and 25 received satisfactions with POGO II. Remaining subjects received satisfaction only after fine tuning the gain value and recommended gains are stored in adaptive expert system. Subsequently, based on the suggested value of gains and additional data from expert audiologists, gain formula could be made distinct for every language.
\end{abstract}

Keywords: Expert System, Audiometer, Frequency, Hearing Loss, Speech Intelligibility, Pure Tone Average, Real Ear Insertion Gain

\section{INTRODUCTION}

Human hearing may be deteriorated because of different reasons. It has been firmly confirmed that noise is the first and important cause for the deterioration of hearing level (Malcovati, 2001). Almost all the human population some or otherwise exposed to different types of noise in our day today life. In general, the deterioration in hearing capability starts as early as in the age of 20s (Nakamura, 2004). The hearing loss is normally due to problems in the middle ear or inner ear. Inner ear problems are usually corrected by fixing of hearing aid (Nalamwar, 2004) The hearing impaired patients are usually unaware of their deterioration in hearing. So they delay wearing of hearing aids and are used to live with little difficulties. Main objective of audiological investigations using computer based system is to perform them in an efficient manner in arriving to the hearing sensitivity of individuals (Gelfand, 1997). Even if all tests are conducted properly, interpretations of the test results are very important so as to recommend an appropriate value of gain. The gain recommendation facility is available in the proposed system. Hence it provides a better satisfaction among the hearing aid users. The proposed system is effectively carries experts knowledge throughout world. At first, the knowledge domain consisting of the expert's opinion and diagnostic methods are created. Subsequently, provision is given to update the knowledge data base as when required, thus making the system as adaptive.

Corresponding Author: Sadagopan Rajkumar, Department of ECE, Faculty of Information and Communication Engineering, Kings Engineering College, Chennai-602117, India 
In the case of hearing aid usage, gain prescription is a mapping or a formula that uses individual's characteristics in amplification settings. The individual characteristics are usually the hearing threshold, Most Comfortable Level (MCL), Loudness Discomfort Level (LDL), also called as Uncomfortable Level (UCL) and loudness contour data being used for the prescription of gain. Initially, it was possible to program a hearing aid with the purpose of normalizing the loudness over a wide range of levels, so that the hearing aid user will perceive the same loudness as a normal hearing person. Next important consideration during prescription of gain is to maximize speech intelligibility. After the hearing aid has been programmed with the initial recommended gain, fine tuning according to the requirements of the user is usually done. It is desirable to minimize the need for fine tuning because it requires considerable effort from the user as well as from the audiologists. Therefore, it is important that the prescribed gain is as close to the optimal gain of the patient as possible.

Hearing aid fitting includes two phases, one is selection and other phase is evaluation phase (Gatehouse, 1993). There are two types of procedures used in practice for hearing aid selection. They are prescriptive and comparative procedure. The design of prescriptive procedure are based on pure tone thresholds or loudness data directly or indirectly involve all the information required to alleviate hearing impairment like lifestyle and living environment. In a comparative procedure, the hearing aid is selected by comparing some audiological parameters like speech intelligibility, sound quality. It is time consuming procedure and also depends on the knowledge and experience of hearing aid fitter. Even though comparative procedure is time consuming procedure, the expertise knowledge of audiologist in the hearing aid fitting is stored in the expert system for formulating a revised formula for the benefit of the hearing impaired patients.

Digital hearing aid of modern days uses different frequency bands in audible range of sound. REIG is gain required for different frequency ranges of sound in the digital hearing aid and it can be modified so as to get a clear perception of sound by the hearing impaired patients. Integrated Real Ear Measurement improves the accuracy of fitting initially and subsequently, when the hearing aid is fine tuned to better speech intelligibility to the patient (Yanz and Galster, 2008; Yanz et al., 2007). Most of the hearing aid users are comfortable with lesser gain than actually prescribed to them. Gerling states that "prescribing the same gain for all individuals simply because they have the same hearing thresholds will result in inaccuracies of too little as well as too much gain" (Gerling, 1992). Over the past several years many prescriptive fitting formulas have been developed and tested to fit hearing aids. The prescriptive formulas have been developed from providing a single target curve for linear instruments to providing two or more target curves for Wide Dynamic Range Compression (WDRC) hearing aids.

National Acoustics Laboratory of Australia developed NAL formula for hearing aids models with linear circuits (Byrne and Tonisson, 1996; Byrne and Dillon, 1986). With the development of non linear hearing aids, a suitable prescriptive procedure was needed to provide multiple gain curves for different input levels. Depending on the patient's practical feedback and preference, final gain settings will vary from these initial settings. With increase in the number of prescriptive formulae quality of service can be defined as the extent to which a particular fitting procedure will give satisfaction to the hearing aid users. Prescriptive method called NAL-R method, is the best method and extensively tested and validated prescriptive methods for linear amplification because of its successful recommendations (Byrne and Dillon, 1986; Byrne and Cotton, 1988; Byrne et al., 1990). The criterion of the comparative method on speech intelligibility tests is more or less according to the procedure suggested by Carhart (1946).

The advantages and limitations of the various prescriptive fitting approaches can be obtained from different sources (Hawkins, 1992; Cornelisse et al., 1995). In this context, the discussion will focus upon two most frequently used methods for linear instruments, as well as present the recent prescriptive methods especially designed for nonlinear hearing aids, including the NAL and POGO formulae were developed in 1976 and 1983, respectively. They are further updated in 1986 (NAL-R) and 1988 (POGO II), to get greater accuracy with the linear instruments (McCandless, 1994; Cox, 1995). The increased use of conventional and programmable nonlinear hearing aid circuits requires new prescriptive methodologies to consider nonlinearity in hearing aids. They further assist clinicians in their ability to fit these products easily and accurately. There are further formulae were created using these approaches are DSL I/O (Byrne et al., 1990), Ihaff (1994) and (Killion, 1995; Kirkwood, 2006; Smeds et al., 2006).

\section{MATERIALS AND METHODS}

\subsection{NAL-R Formula Modifications for Severe to Profound Losses}

The NAL was first formulated by Byrne and Tonisson (1996) and later revised by Byrne and Dillon (1986) as an attempt to prescribe a frequency response 
that gives a clear understanding of speech even if the hearing aid user adjusted the volume control level to their satisfaction. Speech optimization involved an attempt to maximize the speech signal, averaged over a wide frequency range. This procedure did not amplify all speech bands to equal loudness at a comfortable listening level. It is customary to provide too little low frequency gain relative to the mid and high frequency gain. To overcome this difficulty and add more benefits and applications to the NAL procedure, Byrne and Dillon (1986) proposed a revised version of the National Acoustics Laboratory procedure, the NAL-R.

\subsection{Features of NAL-R Formula}

The specific NAL-R formula for the calculation of Real-Ear Insertion Gain (REIG) is presented in Table $\mathbf{1 .}$ The NAL formula includes $10 \mathrm{~dB}$ reserve gain (Mueller, 1992). Two other modifications to the NAL-R formula have been suggested for those with severe sensorineural hearing impairment (Byrne et al., 1990; 1991). Modification 1 is an increase in the A factor equation if the hearing threshold average measured at 500, 1000 and $2000 \mathrm{~Hz}$ exceeds $60 \mathrm{~dB}$ (14). The second modification arises if the degree of hearing threshold at $2000 \mathrm{~Hz}$ exceeds $90 \mathrm{~dB}$. Based on the hearing threshold value this modification suggests an increases the gain in the low frequencies and reduction in the gain for high frequencies. This adjustment alters the hearing aid response of a person with severe hearing loss who requires more low frequency energy for power and less high frequency energy for decreasing the feedback problems (Cox et al., 1994; Dillon, 2001).

\section{Modification 1}

If sum of hearing threshold at 500,1000 and $2000 \mathrm{~Hz}$ exceeds 180 , then $0.116(\mathrm{~S}-180)$ is added to the A Factor where S is the combined total of HL 500, 1000 and 2000.

\section{Modification 2}

If the threshold measured at $2 \mathrm{KHz}$ exceeds $90 \mathrm{~dB}$ then following value is added with the gain value as given in Table 2.

\subsection{POGO II Formula for Calculating Real Ear Gain}

Gain preferences of persons with hearing impairment are very much considered in designing the POGO formula (McCandless and Lyregaard, 1993). Presented gain and output as critical characteristics of the prescription is the additional concern (Cox et al., 1994). Essentially, POGO is Lybarger's 1/2 Gain Rule, with a correction factor suggested for low frequencies to enhance the speech understanding ability. An improved version of this method called POGO II, modifies the gain if the hearing loss at a particular frequency is greater than $65 \mathrm{~dB} \mathrm{HL}$, then the gain is increased by half of the amount that the hearing loss that exceeds $65 \mathrm{~dB}$ explained in step 2 (Hawkins, 1992):

Step1: To compute the formula, the first step is to divide the minimum threshold of hearing at the particular frequency by 2 and record the product as given in Table $\mathbf{3}$.

Step2: Consider whether the hearing loss at a particular frequency being calculated is greater than $65 \mathrm{~dB}$. If it is greater than $65 \mathrm{~dB}$, subtract $65 \mathrm{~dB}$ from the hearing threshold, then half of it is added that factor to the value calculated in step 1. Otherwise the gain is calculated as explained in step 1. Many other prescriptive formulae are used in calculating the gain but among them NAL and POGO-II formulae used popularly because of their success ratio.

Table 1. NAL-R formula for real ear insertion gain

\begin{tabular}{ll}
$\begin{array}{l}\text { Frequency } \\
(\mathrm{HZ})\end{array}$ & Formula + conversion factor = REIG \\
\hline 250 & $\mathrm{~A}+0.31 *$ Hearing Threshold @ 250Hz-17 = REIG \\
500 & $\mathrm{~A}+0.31 *$ Hearing Threshold @ 500Hz-8 = REIG \\
1000 & $\mathrm{~A}+0.31 *$ Hearing Threshold @ $1000 \mathrm{~Hz}-3=$ REIG \\
1500 & $\mathrm{~A}+0.31 *$ Hearing Threshold @ $1500 \mathrm{~Hz}+1=$ REIG \\
2000 & $\mathrm{~A}+0.31 *$ Hearing Threshold @ 2000Hz+1 = REIG \\
3000 & $\mathrm{~A}+0.31 *$ Hearing Threshold @ 3000Hz-1 = REIG \\
4000 & $\mathrm{~A}+0.31 *$ Hearing Threshold @ 4000Hz-2 = REIG \\
6000 & $\mathrm{~A}+0.31 *$ Hearing Threshold @ 6000Hz-2 = REIG
\end{tabular}

Where A = 0.05 (HL @ 500Hz + HL @ 1000Hz + HL @ 2000Hz)

Table 2. NAL-R adjustment for hearing losses when the hearing threshold at $2 \mathrm{KHz}$ exceeds $90 \mathrm{db}$

\begin{tabular}{llllllllll}
\multicolumn{1}{c}{ hearing threshold at $2 \mathrm{KHz}$ exceeds $90 \mathrm{db}$} \\
$\begin{array}{l}\mathrm{HL} \text { at } 2 \mathrm{kHz} \\
\text { dB HL }\end{array}$ & 0.25 & 0.5 & 0.75 & 1 & 1.5 & 2 & 3 & 4 & 6 \\
\hline 95 & 4 & 3 & 1 & 0 & -1 & -2 & -2 & -2 & -2 \\
100 & 6 & 4 & 2 & 0 & -2 & -3 & -3 & -3 & -3 \\
105 & 8 & 5 & 2 & 0 & -3 & -5 & -5 & -5 & -5 \\
110 & 11 & 7 & 3 & 0 & -3 & -6 & -6 & -6 & -6 \\
115 & 13 & 8 & 4 & 0 & -4 & -8 & -8 & -8 & -8 \\
120 & 15 & 9 & 4 & 0 & -5 & -9 & -9 & -9 & -9 \\
\hline
\end{tabular}

Table 3. POGO gain calculations

\begin{tabular}{ll}
\hline Frequency $(\mathrm{HZ})$ & Formula + Conversion factor $=$ REIG \\
\hline 250 & REIG $=1 / 2 \mathrm{HL}+1 / 2(\mathrm{HL}-65)-10$ \\
500 & REIG $=1 / 2 \mathrm{HL}+1 / 2(\mathrm{HL}-65)-5$ \\
750 & REIG $=1 / 2 \mathrm{HL}+1 / 2(\mathrm{HL}-65)$ \\
1000 & REIG $=1 / 2 \mathrm{HL}+1 / 2(\mathrm{HL}-65)$ \\
2000 & REIG $=1 / 2 \mathrm{HL}+1 / 2(\mathrm{HL}-65)$ \\
3000 & REIG $=1 / 2 \mathrm{HL}+1 / 2(\mathrm{HL}-65)$ \\
4000 & REIG $=1 / 2 \mathrm{HL}+1 / 2(\mathrm{HL}-65)$ \\
6000 & REIG $=1 / 2 \mathrm{HL}+1 / 2(\mathrm{HL}-65)$ \\
\hline
\end{tabular}

Peak SSPL $90=[($ UCL @ 500 +1 K + 2K)/3] + 4 
Required rise in $\mathrm{dB}=[$ Minimum threshold of hearing * 0.5].

If the minimum threshold of hearing is greater than $65 \mathrm{~dB}$ then the required rise in $\mathrm{dB}=[$ Minimum threshold of hearing *0.5+0.5 (Minimum threshold of hearing-65)].

These two formulae are been widely used in calculating gain values for the digital hearing aid. It is inbuilt in the adaptive expert system and based on the dissatisfaction from the hearing aid users side the gain is varied to give a clear perception of sound. The recommended gain values are stored to redefine the gain formula.

\subsection{System Description}

The types of audiometric test used to assess the performance of the entire auditory system are pure tone audiometric test and speech audiometric test. In the pure tone audiometric test, the tone used to test the patient with the tone frequency ranging from $125 \mathrm{~Hz}$ to $8000 \mathrm{~Hz}$ are generated with the help of Matlab software so that the accuracy of the test tone is very high. Similarly, the test words used to test the patient in speech audiometric test are also recorded and stored as wave file.

The patient is either recommended for fixing of hearing aid or suitable clinical solutions. If the patient is recommended for hearing aid, then the gains for different frequencies are also displayed. If the recommended gains satisfy the patient, then the values are stored in the data base. If the patient is not satisfied then the audiologist has to sit with the patient and make a number of trials to change the value of gain resulting in a better satisfaction level of the patient with the hearing aid performance. The values of gains which satisfy the patient are being stored in the data base on the runtime thus making this proposed system an adaptive system for audiologists. The suggested values of gain by the audiologists for different aged patients are subsequently analyzed by the system, which will be used to fine tune the formulae for calculation of gain:

Step 1: If the user wants to perform audiometric test, he or she should enter his or her personal data in the patient data screen.

Step 2: If the user wants to see or verify any relevant data or information regarding the audiometric tests they may select appropriate option.

Step 3: In the audiometric test option, at first the user has to select pure tone audiometric test.

Step 4: After finishing pure tone audiometric test the user has to perform speech audiometric test. If both these test results fail to predict comparable results go to step 3 .

Step 5: Based on these test results and data the gain recommendations based on the standard prescriptive procedures are made.

Step 6: If the recommendation doesn't provides satisfaction to the hearing aid users, then the audiologist has to sit with patient and make necessary changes in the gain to enable successful usage of hearing aid.

Step 7: With these values of gain if the patient is satisfied then it is stored in the data base. This will be used in due course for successful fine tuning of the formula.

\subsection{Pure Tone Audiometric Test without Masking}

Pure tone audiogram test includes air-conduction test and bone-conduction test. The purpose of airconduction test is to find the hearing sensitivity at various frequencies. Matlab program generates the test signals with the corresponding $\mathrm{dB}$ ranges from -10 to $+110 \mathrm{~dB}$ and different frequencies 125, 250, 500, 1000, $1500,2000,3000,4000,6000$ and $8000 \mathrm{~Hz}$ for pure tone audiometric test. The average of minimum threshold of hearing for 500, 1000 and $2000 \mathrm{~Hz}$ is known as Pure Tone Average (PTA) which is an important parameter used to predict the hearing level of the tested subject.

\subsection{Pure Tone Audiometric Test WITH Masking}

Pure tone audiometric test with masking is done only when the difference between the air conduction threshold of bad ear with that of bone conduction threshold of good ear is greater than or equal to $10 \mathrm{~dB}$ gets attenuated. The loss of sound energy during the air conduction test, when the stimulus is passing from test ear to the cochlea of the non-test ear is called as Interaural Attenuation (IA) and it ranges from 45 to $80 \mathrm{~dB}$.

Cross hearing is a serious concern in case of bone conduction test than it is for air conduction because both the cochleae are equally stimulated. Therefore to get a reliable test results the non-test ear is not to be involved in the testing procedure by delivering suitable noise signal to it. The masking noise should be loud enough to prevent the tone reaching and stimulating the non-test ear, but at the same time it should not mask the signal given to the test ear which may be called as over masking (Stach, 1998).Thus, an audiologist should select appropriate masking signal level in $\mathrm{dB}$. The pure tone signal is in blue color and masking signal is in red color. 


\subsection{Speech Audiometric Test}

The standard test words are fed to the people at regular intervals and the subject has to identify the words correctly by repeating the words. Based on the number of words correctly identified with the total number of words presented the Speech Discrimination Score (SDS) is obtained. It is to be noted that there is a predictable relationship between the patient's PTA and SDS. Speech audiometry may therefore be useful for validating the results of pure tone audiogram.

\subsection{Recommendations of REIG}

The pure tone thresholds of various frequencies are used for the calculation of real ear insertion gain. Initially it is based on the standard formulae stored in the expert system. If the subject doesn't get satisfaction with the recommendations the audiologist has to change the gain settings with their experience till the satisfaction of the patient. After their satisfactions the gain settings are saved in the data base. This data will be very much useful in developing a standard formula for every language.

\section{RESULTS}

In four different speciality hospitals 272 subjects were tested for prediction of hearing loss using proposed adaptive expert system and also with conventional audiometer model 2001 Digital clinical diagnostic audiometer from Arphi Electronics under same testing conditions. The subjects are also tested with the speech audiometric test using the computerized audiometer and SDS is calculated. The audiograms taken by both modalities are plotted for a subject with moderately severe conductive hearing loss being shown in Fig. 1. The results of the computerized audiometer inbuilt in the adaptive expert system is marked in thick green color whereas conventional audiometer readings are marked in thin red color.

\subsection{Calculation of REIG}

The minimum threshold of hearing obtained with the pure tone audiogram test is used to calculate the REIG for digital hearing aid. If the patient is not suitable to use hearing aid they may be recommended for medical solutions like medicines, surgery. The REIG calculated using NAL-R formula is given in Table 4.

Audiometric thresholds of above mentioned individual:

- Frequency: 250500100015002000300040006000
- Threshold: 5045656570605045

- Step 1: X = 0.15 (Hearing threshold @500+@1000 $+@ 2000) / 3$

- $\mathrm{X}=0.15(45+65+70) / 3$

- $\mathrm{X}=9$

The same patient's hearing aid can be programmed with the help of gain recommendations calculated using the POGO II formula. The prescription of real ear insertion gain using POGO II formula is calculated and shown in Table 5. A low frequency factor of $-10,-5 \mathrm{~dB}$ is added for 250 and $500 \mathrm{~Hz}$ respectively (Martin, 2008; Schwartz et al., 1988).

The patient is not satisfied with recommendations of REIG for various frequencies using POGO II formula and NAL-R formula and hence the expert audiologist makes some corrections in the gain settings for arriving to the satisfaction of the subject concerned and the gain recommendations of the standard formulae and expert audiologist recommendations are given in Table 6.

The recommendations of REIG for various frequency ranges using POGO II formula and NAL-R formula for the same test results of a patient and also final suggestions by the audiologist are plotted as shown in Fig. 2.

Similar type of gain calculations and gain suggestions for a subject with mild hearing loss is being compensated by careful alignment of the gain by expert audiologist to give a clear speech perception is given in Table 7 and the value is plotted as shown in Fig. 3 .

Table 4. NAL-R real ear insertion gain calculations

\begin{tabular}{llll}
\hline $\begin{array}{l}\text { Frequency } \\
(\mathrm{Hz})\end{array}$ & Formula & $\begin{array}{l}\text { Conversion } \\
\text { factor }\end{array}$ & REIG \\
\hline 250 & $9+0.31(50)=24.25$ & -17 & 7.25 \\
500 & $9+0.31(45)=22.95$ & -8 & 14.95 \\
1000 & $9+0.31(65)=29.15$ & -3 & 26.15 \\
1500 & $9+0.31(65)=29.15$ & +1 & 30.15 \\
2000 & $9+0.31(70)=30.70$ & +1 & 31.70 \\
3000 & $9+0.31(60)=27.60$ & -1 & 26.60 \\
4000 & $9+0.31(50)=24.50$ & -2 & 25.50 \\
6000 & $9+0.31(45)=22.95$ & -2 & 20.95 \\
\hline
\end{tabular}

Table 5. POGO-II real ear insertion gain calculations

\begin{tabular}{llll}
\hline Frequency $(\mathrm{Hz})$ & Formula & Conversion factor & REIG \\
\hline 250 & $25.0+0$ & -10 & 15.0 \\
500 & $22.5+0$ & -5 & 17.0 \\
1000 & $32.5+0$ & 0 & 32.5 \\
1500 & $32.5+0$ & 0 & 32.5 \\
2000 & $35.0+2.5$ & 0 & 37.5 \\
3000 & $30.0+0$ & 0 & 30.0 \\
4000 & $25.0+0$ & 0 & 25.0 \\
6000 & $22.5+0$ & 0 & 22.5 \\
\hline
\end{tabular}




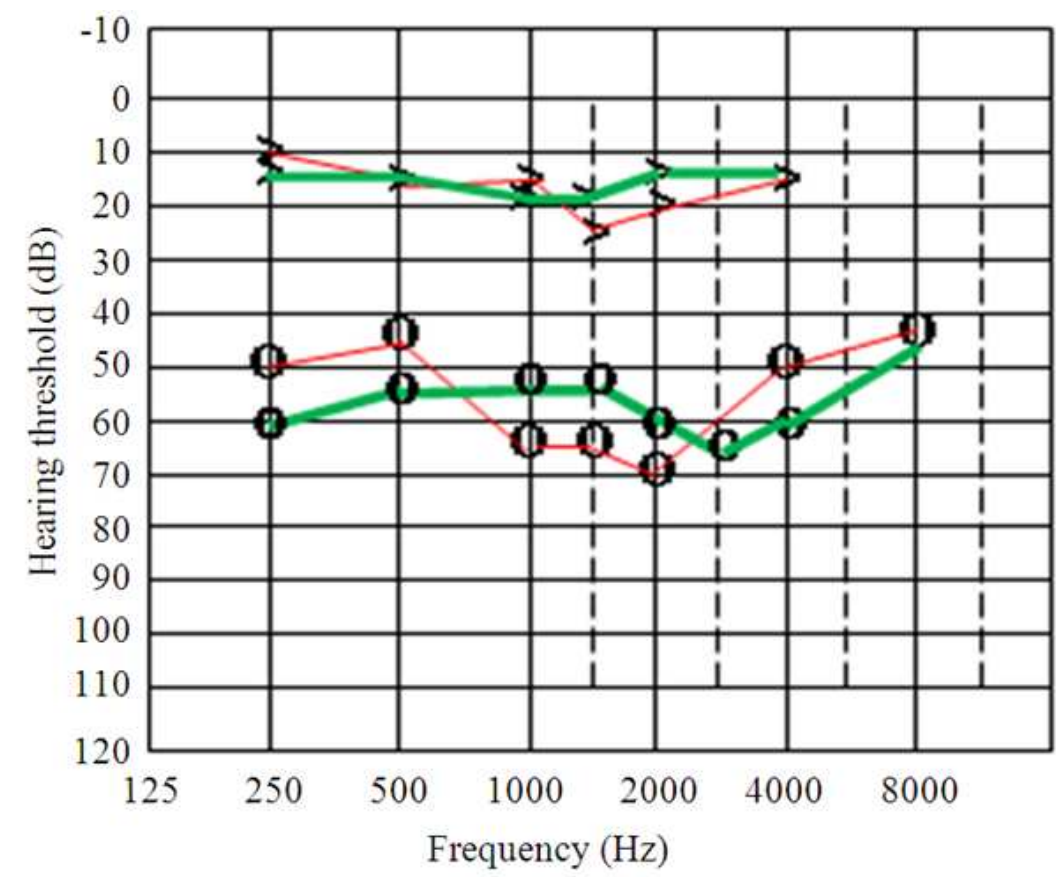

$>$-- Bone conduction threshold

$\mathrm{O}$-- Air conduction threshold

-- Conventional audiometer reading

-- Computerized audiometer reading

Fig. 1. Audiograms of a patient with conductive hearing loss

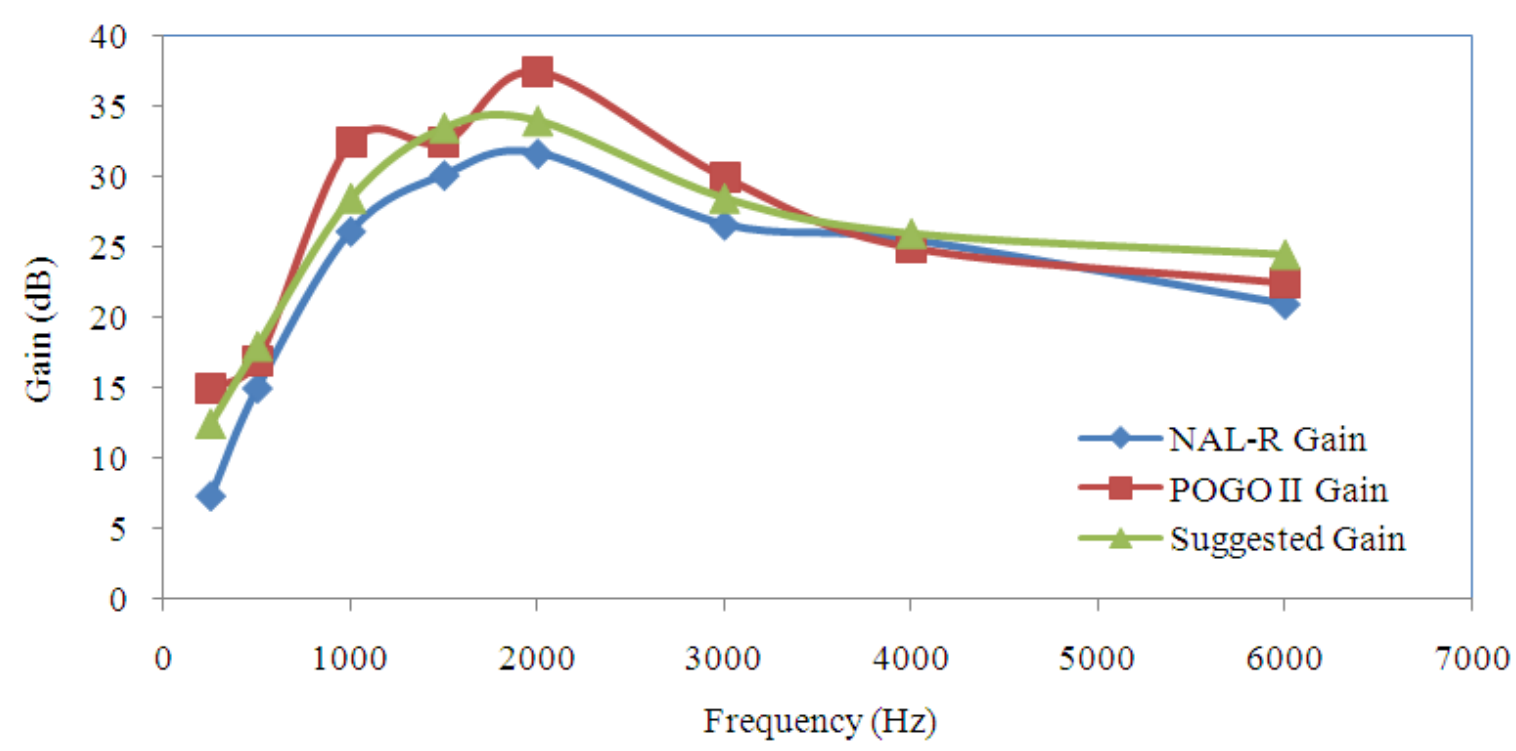

Fig. 2. POGO-II and NAL-R and the suggested recommendations of REIG 


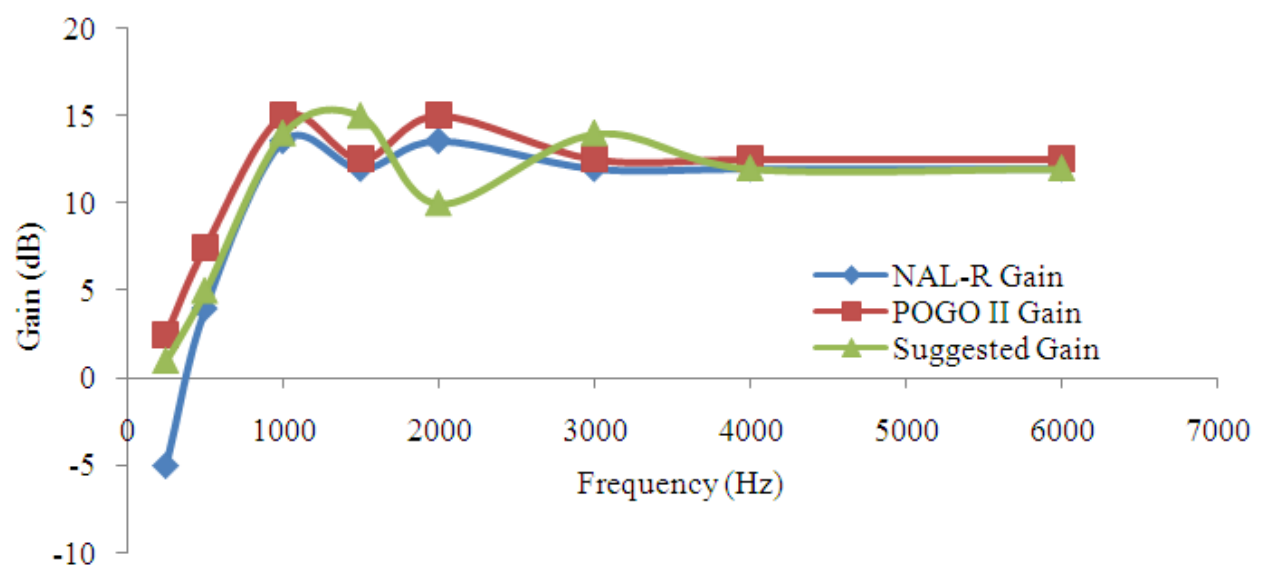

Fig. 3. Gain suggestion for subject with mild hearing loss

Table 6. Real ear insertion gain suggestions of standard formulae and expert audiologist

\begin{tabular}{llll}
\multicolumn{5}{c}{ formulae and expert audiologist } \\
\hline $\begin{array}{l}\text { Frequency } \\
(\mathrm{Hz})\end{array}$ & $\begin{array}{l}\text { NAL-R } \\
\text { Gain }\end{array}$ & $\begin{array}{l}\text { POGO } \\
\text { II Gain }\end{array}$ & $\begin{array}{l}\text { Suggested } \\
\text { gain }\end{array}$ \\
\hline 250 & 7.25 & 15.0 & 12.5 \\
500 & 14.95 & 17.0 & 18.0 \\
1000 & 26.15 & 32.5 & 28.5 \\
1500 & 30.15 & 32.5 & 33.5 \\
2000 & 31.70 & 37.5 & 34.0 \\
3000 & 26.60 & 30.0 & 28.5 \\
4000 & 25.50 & 25.0 & 26.0 \\
6000 & 20.95 & 22.5 & 24.5 \\
\hline
\end{tabular}

Table 7. Gain suggestions for a subject with mild hearing loss

\begin{tabular}{llll}
\hline $\begin{array}{l}\text { Frequency } \\
(\mathrm{Hz})\end{array}$ & $\begin{array}{l}\text { NAL-R } \\
\text { Gain }\end{array}$ & $\begin{array}{l}\text { POGO } \\
\text { II Gain }\end{array}$ & $\begin{array}{l}\text { Suggested } \\
\text { gain }\end{array}$ \\
\hline 250 & -5.0 & 2.50 & 1.00 \\
500 & 4.00 & 7.50 & 5.00 \\
1000 & 13.6 & 15.0 & 14.0 \\
1500 & 12.0 & 12.5 & 15.0 \\
2000 & 13.6 & 15.0 & 10.0 \\
3000 & 12.0 & 12.5 & 14.0 \\
4000 & 12.0 & 12.5 & 12.0 \\
6000 & 12.0 & 12.5 & 12.0 \\
\hline
\end{tabular}

\section{DISCUSSION}

Comparison of test results taken with the audiometer inbuilt in the expert system and conventional audiometer can be done using Fig. 1. The pure tone average arrived using computerized audiometer is 56.66 whereas it is 60 when the same subject is tested with conventional audiometer. The interpretation of computerized audiometer results is same as that of conventional audiometer results except with the marginal difference in the value of PTA.
The gain calculations for all the 127 subjects were analyzed. In almost all cases the variation required for high frequency ranges as against the gain suggested by the system is very less. In the low frequency region mild variation is required. In the mid frequency region where majority of the speech frequencies available much variation in the suggested gain is required. It has been compared two different types of hearing-aid fitting procedures in a double-blind randomized clinical study i.e., hearing aid fittings based on NAL-R formula and POGO II formula. Main outcome measures were improvement of speech intelligibility scores in quiet and noise conditions. Data were related to the real ear insertion responses that were measured after fitting. For analysis purposes subgroups were composed according to degree of hearing loss, characterized by unaided speech intelligibility in quiet, previous experience with hearing aids, unilateral or bilateral fittings and a type of hearing aid.

We found equal improvement of speech intelligibility in quiet, while fitting according to the NAL-R formula resulted in a somewhat better performance as expressed by the speech-to-noise ratio in comparison to the POGO II formula. Both procedures resulted in comparable real-ear insertion responses. Both these formulae were tested with 127 patients found to be suitable to use wearing aid for arriving at an optimum formula used to calculate the Real Ear Insertion Gain to get a satisfactory performance with the digital hearing aid. Of these, 28 subjects had satisfaction with NAL-R formula and 25 subjects were satisfied with POGO II recommendations. Remaining people received satisfaction only after adjusting gain settings by experienced audiologists. These successful gain settings were stored in data base for formulating a revised 
formula to give complete satisfaction among the hearing aid users irrespective of their age, sex and living environment. The system will give an adaptive solution for all these kind of variations because it is developed with the feedback received from the patient side.

\section{CONCLUSION}

Adaptive Expert System for the calculation of real ear insertion gain was designed and developed with the valuable suggestions from expert audiologists of different hospitals. Audiological tests are being carried out with the help of this set up and the audiograms, the calculated value of speech discrimination score are stored for future references. All subjects were also tested with conventional audiometer 2001 Digital clinical diagnostic audiometer from Arphi Electronics and also with computerized audiometer inbuilt in the expert system under ideal conditions. The accuracy of the proposed expert system was found to be $94.87 \%$. If any correction made by the experienced audiologists in the gain values suggested by the expert system are also stored for fine tuning the REIG formula. Subsequently, this expert knowledge domain has been used in the calculation of REIG. This would not only reduce the workload of the audiologist but also leads to recommendation of superior technical specifications with regard to the design of hearing aid. This additional specialized attention certainly would bring better satisfaction among the hearing aid users. This expert system enables audiologists to perform testing procedure in a standard sequence and recommend most accurate REIG suggestions so that the hearing impaired person gets satisfied with the performance of the hearing aid. The different types of hearing loss suffered by the world population and successful gain suggestions have to be analyzed with the expert system for further fine tuning of the formula used for calculating REIG. The formula can be made distinctive to any language.

\section{REFERENCES}

Byrne, D. and H. Dillon, 1986. The National Acoustic Laboratories' (NAL) new procedure for selecting the gain and frequency response of a hearing aid. Ear. Hear., 7: 257-265. PMID: 3743918

Byrne, D. and S. Cotton, 1988. Evaluation of the National Acoustic Laboratories' new hearing aid selection procedure. J. Speech Hear Res., 31: 178186. PMID: 3398491
Byrne, D. and W. Tonisson, 1996. Selecting the gain of hearing aids for persons with sensorineural hearing impairments. Scand Audiol., 5: 51-59. DOI: 10.3109/01050397609043095

Byrne, D., A. Parkinson and P. Newell, 1991. Modified hearing aid selection procedures for severe/profound hearing losses. In: The Vanderbilt hearing aid report II, Stude-baker, G.A., F.H. Bess and L.B. Beck, Eds., York Press, Incorporated, ISBN-10: 0912752262, pp: 295-300.

Byrne, D., A. Parkinson and P.H. Newall, 1990. Hearing aid gain and frequency response requirements for the severely/profoundly hearing impaired. Ear. Hear, 11: 40-49. PMID: 2307302

Carhart, R., 1946. Tests for selection of hearing aids. Laryngoscope, 56: 780-794. DOI: 10.1288/00005537-194612000-00004

Cornelisse, L.E., R.C. Seewald and D.G. Jamieson, 1995. The input/output formula: A theoretical approach to the fitting of personal amplification devices. J. Acoust. Soc. Am., 97: 1854-64. PMID: 7699167

Cox, R.M., 1995. Using loudness data for hearing aid selection: The IHAFF approach. Hear J., 48: 39-44.

Cox, R., C.M. Goff, S.E. Martin and L.L. McLoud, 1994. The contour test: Normative data. University of Memphis.

Dillon, H., 2001. Prescribing Hearing Aid Performance.

Gatehouse, S.,1993. Hearing aid evaluation: Limitations of present procedures and future requirements. JSLPA Monogr Suppl1, 17: 50-57.

Gelfand, A.S., 1997. Essentials of Audiology, 2nd Edn., Thieme Medical Publishers, Incorporated, New York, ISBN-10: 0865776210, pp: 562.

Gerling, I.J., 1992. Evaluation of prescriptive fitting. J. Am. Acad. Audiol., 3: 331-337. PMID: 1421469

Hawkins, D., 1992. Prescriptive approaches to selection of gain and frequency response.

Ihaff, 1994. A comprehensive hearing aid fitting protocol.

Killion, M., 1995. Talking hair cells: What they say about hearing aids. Thesis, Ph.D., San Diego, CA.

Kirkwood, D., 2006. Survey: Dispensers fitted more hearing aids in 2005 at higher prices. Hearing J., 59: 48-48.

Malcovati, P., 2001. Design of integrated circuits for audiometric applications. IEEE Int. Symp. Circ. Syst., $\quad 2$ : $1.7 .1-1.7 .14$ DOI: 10.1109/TUTCAS.2001.946902 
Martin, F.N., 2008. Introduction to Audiology. 1st Ed., Pearson Education India, ISBN-10: 8131724735, pp: 502.

McCandless, G. and P. Lyregaard, 1983. Prescription of Gain and Output (POGO) for hearing aids. Hear. Instr., 34: 16-21.

McCandless, G., 1994. Overview and rationale of threshold basedhearing aid selection procedures. In: Strategies for selecting and verifying hearing aid fittings, Valente, M. (Ed.). Thieme-Stratton New York, ISBN-10: 0865775001.

Mueller, H.G., 1992. Individualizing the ordering of custom hearing aids, In: Probe-Microphone Measurements: Hearing Aid Selection and Assessment, Mueller, H.G., D.B. Hawkins, J.L. Northern, Eds., Singular Pub. Group, San Diego, ISBN-10: 1879105683, pp: 297-297.

Nakamura, N., 2004. Development of mobile audiometric test system using mobile phones. Proceedings of the IEEE EMBS Asian-Pacific Conference on Biomedical Engineering, Oct. 20-22, IEEE Xplore Press, pp: 3369-3372. DOI: 10.1109/APBME.2003.1302731
Nalamwar, M.K., 2004. Advanced audiometer a novel signal generator technique.

Schwartz, D., P. Lyregaard and P. Lundh, 1988. Hearing aid selection for severe-to-profound hearing loss. Hear J., 41: 13-17.

Smeds, K., G. Keidser, J. Zakis, H. Dillon and A. Leijon et al., 2006. Preferred overall loudness. II: Listening through hearing aids in field and laboratory tests. Int. J. Audiol., 1: 12-25. PMID: 16562560

Stach, A., 1998. Clinical audiology: An Introduction. 1st Edn., Cengage Learning, San Diego, ISBN-10: 156593346X, pp: 585.

Yanz, J.L. and J. Galster, 2008. Integrating Real-ear measurement into hearing aid fittings. Adv. Audiologists, 10: 38-44.

Yanz, J.L., J.F.D. Pisa and L. Olson, 2007. Integrated REM: Real-ear measurement from a hearing aid. Hearing Rev., 14: 44-51. 